\title{
Efficient Allocation of Testing Resources for Software Module Testing Based on the Hyper-Geometric Distribution Software Reliability Growth Model
}

\author{
Rong-Huei Hou \& Sy-Yen Kuo \\ Department of Electrical Engineering \\ National Taiwan University \\ Taipei, Taiwan, R.O.C. \\ Email: sykuo@cc.ee.ntu.edu.tw
}

\author{
Yi-Ping Chang \\ Department of Business Mathematics \\ Soochow University \\ Taipei, Taiwan, R.O.C.
}

\begin{abstract}
Considerable amount of testing resources is required during software module testing. In this paper, based on the Hyper-Geometric Distribution software reliability growth Model (HGDM) we investigate the following two optimal resource allocation problems in software module testing: 1) minimization of the number of software faults still undetected in the system after testing given a total amount of testing resources, and 2) minimization of the total amount of testing resources required given the number of software faults still undetected in the system after testing. Furthermore, based on the concepts of average allocation and proportional allocation, two simple allocation methods are also introduced. Experimental results show that the optimal allocation method can improve the quality and reliability of the software system much more significantly than the simple allocation methods can. Therefore, the optimal allocation method is very efficient for solving the testing resource allocation problem.
\end{abstract}

\section{Introduction}

A software system usually consists of many complex software modules, and the software testing phase is divided into three successive stages [1]: module testing, integration testing, and system testing. Considerable amount of testing resources is required during software module testing. These resources are man-power, CPU time, number of test cases, etc. To develop a quality and reliable software system, a project manager has to determine in advance the optimal way to allocate the testing resources for each module [2-4].

In literature, many Software Reliability Growth Models (SRGMs) which describe the relationship between the cumulative number of detected faults and the duration of the testing have been proposed [5-
7]. The Hyper-Geometric Distribution software reliability growth Model (HGDM) was first proposed by Tohma et al. [8]. A series of studies on the HGDM have been made recently [9-14].

In this paper, based on the HGDM with logistic learning factor [12] we investigate two optimal resource allocation problems in software module testing: 1) minimization of the number of software fault$s$ still undetected in the system after testing given a total amount of testing resources, and 2) minimization of the total amount of testing resources required given the number of software faults still undetected in the system after testing. Two efficient and novel optimization algorithms based on the Lagrange multiplier method [15] for the above two problems will be proposed, respectively. Furthermore, based on the concepts of average allocation and proportional allocation, two simple allocation methods, the average allocation method and the proportional allocation method, will also be introduced, respectively. Experimental results show that the optimal allocation methods can improve the quality and reliability of the software system much more significantly than the simple allocation methods can. Therefore, the optimal allocation methods are very efficient for solving the testing resource allocation problem.

The organization of this paper is as follows. Section 2 briefly reviews the HGDM with logistic learning factor. Based on this model, two optimal resource allocation problems in software module testing are discussed in Section 3. The relationship between the optimal, average, and proportional resource allocation methods is investigated in Section 4. Numerical examples are presented for comparison in Section 5 followed by the conclusions in Section 6 .

\section{Notations}

$M \quad$ number of software modules 
$m_{j}, m$ expected number of initial software faults in module $j$ and the whole system, respectively

$t_{i} \quad$ the $i^{t h}$ test instance, $i=1,2, \ldots$ where $i$ represents the order of application

$w_{i} \quad$ number of faults newly detected or redetected by $t_{i}$

$q_{j}, Q \quad$ amount of testing resources allocated to module $j$ and the whole system during the application of $t_{k}$, respectively

$\mathcal{Q} \quad\left(q_{1}, q_{2}, \ldots, q_{M}\right) ;$ vector of $q_{j}$

$I(A) \quad \begin{cases}1, & \text { if } A \text { occurs } \\ 0, & \text { otherwise }\end{cases}$

$p_{L T, j}, a_{j}, b_{j}$ parameters of the HGDM with logistic learning factor in module $j$

$p_{j, i} \quad p_{L T, j} I\left(q_{j}>0\right) /\left(1+e^{-q_{j}\left(a_{j} i+b_{j}\right)}\right), j=1,2, \ldots, M$

$z_{j}, Z$ expected number of software faults undetected in module $j$ and the whole system after the application of $t_{1}, t_{2}, \ldots, t_{k}$, respectively

$v_{j} \quad$ weighting factor for module $j$

$m_{j}^{*} \quad m_{j} \prod_{i=1}^{k-1}\left(1-p_{j, i}\right)$

$C_{j, k}, C_{k}$ number of faults in module $j$ and the whole system detected after the application of $t_{1}, t_{2}, \ldots, t_{k}$, respectively

$a_{j}^{*} \quad a_{j} k+b_{j}, j=1,2, \ldots, M$

$A_{j} \quad v_{j} m_{j}^{*} p_{L T, j} a_{j}^{*}, j=1,2, \ldots, M$

$B_{j} \quad e^{-a_{j}^{*} q_{j}}, j=1,2, \ldots, M$

$\lambda$ Lagrange multiplier

$L(\mathcal{Q}, \lambda)$ Lagrangean

\# optimal solution of the resource allocation problem

$q_{j}^{\#}, q_{j, \text { avg }}, q_{j, \text { prop }}$ amount of testing resources allocated to module $j$ during the application of $t_{k}$ by the optimal, average, and proportional allocation methods, respectively

$Z_{\text {opt }}, Z_{\text {avg }}, Z_{\text {prop }}$ number of software faults still undetected in the whole system estimated by the optimal, average, and proportional allocation methods, respectively

\section{Assumptions}

1. A software system is composed of $M$ modules and each module is tested independently.

2. For each module, faults already detected so far by $t_{1}, t_{2}, \ldots, t_{k-1}$ have been collected. Based on these faults, the parameters of each module, $m_{j}$, $p_{L T, j}, a_{j}$, and $b_{j}$ for $j=1,2, \ldots, M$, can be estimated [12].

3. The manager has to decide how to allocate the testing resources to each module during the application of test instance $t_{k}$.

\section{HGDM with Logistic Learning Factor}

In this section, we briefly review the HGDM [8-

10]. At the beginning of the test/debug stage, $m$ initial faults are resident in the program. With the application of "test instances", faults can be detected by the testers. The collection of test operations performed in a unit of time (one day, one week, ...) is called a "test instance". At the end of a test instance $t_{i}$, each fault will be classified into one of the following two categories, newly detected faults or redetected faults. Some of the faults detected by $t_{i}$ may have already been detected by the application of $t_{1}, t_{2}, \ldots, t_{i-1}$. Therefore, the number of faults newly detected by $t_{i}$ is not necessarily equal to $w_{i}$.

The exact development of the model in terms of mathematical equations has been shown in $[8-10]$. The mean value function of the HGDM is given by

$$
E C_{i}=m\left[1-\prod_{j=1}^{i}\left(1-\frac{w_{j}}{m}\right)\right]=m\left[1-\prod_{j=1}^{i}\left(1-p_{j}\right)\right.
$$

with $E C_{0}=0$ where $p_{i}=w_{i} / m, i=1,2, \ldots$.

Various functions for the learning factor $p_{i}$ have been proposed in $[8-10]$. To make the HGDM more realistic and practical, a logistic learning factor based on the S-shaped learning curve was proposed in [12]. The logistic learning factor is

$$
p_{i}=p_{L T} \frac{1}{1+e^{-u_{i}(a i+b)}}, a, b, u_{i}>0,0<p_{L T} \leq 1,
$$

where $u_{i}>0$ represents the testing resource consumed in $t_{i}$. Note that if there is no testing resource used in $t_{i}$ (i.e., $u_{i}=0$ ), obviously no faults can be detected (i.e., $w_{i}=p_{i}=0$ ). Therefore, it is reasonable that Eq.(2) can be modified to be

$$
p_{i}=p_{L T} \frac{I\left(u_{i}>0\right)}{1+e^{-u_{i}(a i+b)}}, i=1,2, \ldots
$$

\section{Two Optimal Resource Allocation Problems}

To improve the quality and reliability of a software system, the manager of software development has to effectively apportion the testing resources among the modules [2-4]. In this section, based on the HGDM with logistic learning factor, two optimal resource allocation problems in software module testing are discussed.

\subsection{Minimizing the number of undetected faults}

In this subsection, we study the following problem. Assuming that the total amount of testing resources for software module testing is given, the manager has to allocate these resources to each module to minimize the number of software faults still undetected in the whole system after the application of $t_{1}, t_{2}, \ldots, t_{k}[2-4]$. 
Let the amount of resources allocated to module $j$ during the application of $t_{k}$ be $q_{j}$, from Eq.(3) it is obvious that

$$
p_{j, k}=p_{L T, j} \frac{I\left(q_{j}>0\right)}{1+e^{-q_{j}\left(a_{j} k+b_{j}\right)}}, a_{j}, b_{j}>0,0<p_{L T, j} \leq 1 .
$$

Let $C_{j, k}$ denote the number of software faults in module $j$ already detected so far by $t_{1}, t_{2}, \ldots, t_{k}$. From Eqs.(1) and (4), the expected value of $C_{j, k}$ denoted by $E C_{j, k}$ is $[8-10]$

$$
\left\{\begin{array}{l}
E C_{j, 0}=0, \\
E C_{j, k}=m_{j}\left[1-\prod_{i=1}^{k}\left(1-p_{j, i}\right)\right], \quad j=1,2, \ldots, M .
\end{array}\right.
$$

Therefore, the number of software faults still undetected in module $j$ after the application of $t_{1}, t_{2}, \ldots, t_{k}$ can be estimated by Eqs. (4) and (5) as:

$$
\begin{aligned}
z_{j}=m_{j}-E C_{j, k} & =\left\{m_{j} \prod_{i=1}^{k-1}\left(1-p_{j, i}\right)\right\}\left(1-p_{j, k}\right) \\
& =m_{j}^{*}\left(1-\frac{p_{L T, j} I\left(q_{j}>0\right)}{1+e^{-q_{j}\left(a_{j} k+b_{j}\right)}}\right) .(6)
\end{aligned}
$$

Note that $m_{j}^{*}$ represents the expected number of software faults still undetected in module $j$ after the application of $t_{1}, t_{2}, \ldots, t_{k-1}$. Suppose the total amount of testing resources for module testing is $Q$, from Eq.(6) this allocation problem can formulated as:

$$
\begin{array}{cl}
\operatorname{Min} & \sum_{j=1}^{M} v_{j} m_{j}^{*}\left(1-\frac{p_{L T, j} I\left(q_{j}>0\right)}{1+e^{-q_{j}\left(a_{j} k+b_{j}\right)}}\right) \\
\text { subject to } & \sum_{j=1}^{M} q_{j}=Q, \\
& q_{j} \geq 0, \quad j=1,2, \ldots, M .
\end{array}
$$

Note that $v_{j}$ is a weighting factor to represent the relative importance of a fault detected from module $j$ in the future. This problem can be easily rewritten in a more compact formulation (called Problem P1):

$$
\begin{array}{ll}
\text { P1: Max } & f(\mathcal{Q})=\sum_{j=1}^{M} \frac{v_{j} m_{j}^{*} p_{L r_{, j}} I\left(q_{j}>0\right)}{1+e^{-q_{j}\left(a_{j} k+b_{j}\right)}}(8) \\
\text { subject to } & \sum_{j=1}^{M} q_{j}=Q \\
& q_{j} \geq 0, j=1,2, \ldots, M .
\end{array}
$$

Ignoring the constraint $q_{j} \geq 0$ and using the Lagrange method [15], Problem $\mathrm{P} 1$ is equivalent to find the maximum of

$$
L_{1}(\mathcal{Q}, \lambda)=\sum_{j=1}^{M} \frac{v_{j} m_{j}^{*} p_{L T, j} I\left(q_{j}>0\right)}{1+e^{-a_{j}^{*} q_{j}}}+\lambda\left(\sum_{j=1}^{M} q_{j}-Q\right) .
$$

For convenience, we consider another problem (called Problem $P 1^{\prime}$ ):

$$
\begin{array}{ll}
\mathrm{P}^{\prime}: \operatorname{Max} & f(\mathcal{Q})=\sum_{j=1}^{M} \frac{v_{j} m_{j}^{*} p_{L T, j}}{1+e^{-q_{j}\left(a_{j} k+b_{j}\right)}} \\
\text { subject to } & \sum_{j=1}^{M} q_{j}=Q, \\
q_{j} \geq 0, j=1,2, \ldots, M .
\end{array}
$$

Ignoring the constraint $q_{j} \geq 0$ and using the Lagrange method [15], Problem $P 1^{\prime}$ is equivalent to find the maximum of

$$
L_{2}(\mathcal{Q}, \lambda)=\sum_{j=1}^{M} \frac{v_{j} m_{j}^{*} p_{L T, j}}{1+e^{-a_{j}^{*} q_{j}}}+\lambda\left(\sum_{j=1}^{M} q_{j}-Q\right) .
$$

According to the Kuhn-Tucker conditions [15], the necessary conditions A1-A3 for a maximum of Eq.(10) to exist are as follows:

$\mathrm{A} 1: \frac{\partial L_{2}(\mathcal{Q}, \lambda)}{\partial q_{j}}=0, j=1,2, \ldots, M$.

A2: $\lambda<0$.

A3: $\sum_{j=1}^{M} q_{j}=Q$.

For Condition A1, from Eq.(10) we have

$$
\frac{\partial L_{2}(\mathcal{Q}, \lambda)}{\partial q_{j}}=\frac{v_{j} m_{j}^{*} p_{L T, j} a_{j}^{*} e^{-a_{j}^{*} q_{j}}}{\left(1+e^{-a_{j}^{*} q_{j}}\right)^{2}}+\lambda=0 .
$$

That is,

$$
\frac{A_{j} B_{j}}{\left(1+B_{j}\right)^{2}}+\lambda=0, j=1,2, \ldots, M
$$

Since $a_{j}^{*}>0$ and $q_{j}$ should be larger than or equal to zero for $j=1,2, \ldots, M$, we have $A_{j}>0$ and $0<B_{j} \leq 1$ for $j=1,2, \ldots, M$. Therefore, from Eq. (12) we have $\lambda<0$ (i.e., Condition A2 is satisfied) and

$$
\frac{B_{j}}{\left(1+B_{j}\right)^{2}}=-\frac{\lambda}{A_{j}} \text {. }
$$

Since $0<B_{j} \leq 1$, from Eq.(13) we can easily obtain

$$
0<\frac{B_{j}}{\left(1+B_{j}\right)^{2}} \leq \frac{1}{4}, j=1,2, \ldots, M .
$$

That is,

$$
-\frac{A_{j}}{4} \leq \lambda<0, j=1,2, \ldots, M .
$$


From Eq.(13), we have

$$
\frac{\lambda}{A_{j}}\left(1+B_{j}\right)^{2}+B_{j}=0 .
$$

Since $0<B_{j} \leq 1$ and $-1 / 4 \leq \lambda / A_{j}<0$, the root of Eq.(16) is

$$
B_{j}=-1-\frac{1-\sqrt{1+4 \lambda / A_{j}}}{2 \lambda / A_{j}} .
$$

Therefore, from Eq.(17) we have

$$
q_{j}=-\frac{1}{a_{j}^{*}} \ln \left(-1-\frac{1-\sqrt{1+4 \lambda / A_{j}}}{2 \lambda / A_{j}}\right) .
$$

Let

$$
\begin{aligned}
S_{L}(\lambda) & =\sum_{j=1}^{L} \frac{1}{a_{j}^{*}} \ln \left(-1-\frac{1-\sqrt{1+4 \lambda / A_{j}}}{2 \lambda / A_{j}}\right) \\
& =\sum_{j=1}^{L} s_{j}(\lambda), L=1,2, \cdots, M,
\end{aligned}
$$

where

$$
s_{j}(\lambda)=\frac{1}{a_{j}^{*}} \ln \left(-1-\frac{1-\sqrt{1+4 \lambda / A_{j}}}{2 \lambda / A_{j}}\right) .
$$

By simple calculation, we have the following lemma.

\section{Lemma 1.}

(i) $s_{j}(\lambda)$ is decreasing in $\lambda$;

(ii) $\lim _{\lambda \rightarrow 0^{-}} s_{j}(\lambda)=-\infty$;

(iii) $\lim _{\lambda \rightarrow-A_{j} / 4} s_{j}(\lambda)=0$, where $s_{j}(\lambda)$ is given in Eq.(21).

Since $S_{L}(\lambda)=\sum_{j=1}^{L} s_{j}(\lambda)$ and $s_{j}(\lambda)$ is decreasing in $\lambda, S_{L}(\lambda)$ is decreasing in $\lambda$ for $L=1,2, \cdots, M$. Since $\lambda \geq-A_{j} / 4$ for $j=1,2, \ldots, M$, for convenience we rearrange the indices of $A_{j}$ 's such that $A_{1} \geq A_{2} \geq$ $\ldots \geq A_{M}$. Moreover, from Eq. (21) we have $s_{j}(\lambda)<0$ for all $j$, and $S_{M}(\lambda)$ and $S_{M-1}(\lambda)$ are shown in Figure 1 , where $\alpha_{M}=\max _{\lambda \geq-A_{M} / 4} S_{M}(\lambda)=S_{M}\left(-A_{M} / 4\right)$ and $\alpha_{M-1}=\max _{-A_{M} / 4>\lambda \geq-A_{M-1} / 4} S_{M-1}(\lambda)=S_{M-1}\left(-A_{M-1} / 4\right)$.

To satisfy the constraint $\sum_{j=1}^{M} q_{j}=Q$ (i.e., Condition $\mathrm{A} 3$ ) and based on Figure 1, the main steps for finding the maximum of Problem P1 is depicted in the following:

Step 1. Rearrange the indices of $A_{j}$ 's such that $A_{1} \geq$ $A_{2} \geq \ldots \geq A_{M}$.
Step 2. (i) If $\max _{\lambda \geq-A_{M} / 4} S_{M}(\lambda)=$

$\sum_{j=1}^{M} \frac{1}{a_{j}^{*}} \ln \left(-1-\frac{1-\sqrt{1-A_{M} / A_{j}}}{-\frac{2}{A_{j}} \frac{A_{M}}{4}}\right) \geq-Q$, there exists a unique root $\lambda^{\#}$ (here, $\lambda^{\#} \geq$ $\left.-A_{M} / 4\right)$ such that $S_{M}\left(\lambda^{\#}\right)=-Q$ and hence we are done. The optimal solution

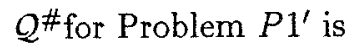

$q_{j}^{\#}=-\frac{1}{a_{j}^{*}} \ln \left(-1 \frac{1-\sqrt{1+4 \lambda^{\# / A_{j}}}}{2 \lambda^{\#} / A_{j}}\right), j=1,2, \ldots, M$.

Since $q_{j}^{\#}>0, j=1,2, \ldots, M$, the optimal solution for Problem $\mathrm{P} 1$ is also $\mathcal{Q}^{\#}$.

(ii) If $\max _{\lambda \geq-A_{M} / 4} S_{M}(\lambda)=$

$\sum_{j=1}^{M} \frac{1}{a_{j}^{*}} \ln \left(-1-\frac{1-\sqrt{1-A_{M} / A_{j}}}{-\frac{2}{A_{j}} \frac{A_{M}}{4}}\right)<-Q$,

we have $\lambda<-A_{M} / 4$ and hence from Eq.(12)

$$
\frac{A_{M} B_{M}}{\left(1+B_{M}\right)^{2}}+\lambda<\frac{A_{M}}{4}-\frac{A_{M}}{4}=0
$$

that is,

$$
\frac{\partial L_{2}(\mathcal{Q}, \lambda)}{\partial q_{M}}<0
$$

Therefore, $L_{2}(\mathcal{Q}, \lambda)$ is decreasing in $q_{M}$. Furthermore, it can be easily shown that $L_{1}(\mathcal{Q}, \lambda)$ is also decreasing in $q_{M}$. To maximize $L_{1}(\mathcal{Q}, \lambda)$, let $q_{M}^{\#}=q_{M}=0$ (since $q_{M} \geq 0$ ) and go to Step 3 .

Step 3. (i) If If $\max _{-A_{M} / 4>\lambda \geq-A_{M-1} / 4} S_{M-1}(\lambda)=$
$\sum_{j=1}^{M-1} \frac{1}{a_{j}^{*}} \ln \left(-1-\frac{1-\sqrt{1-A_{M-1} / A_{j}}}{-\frac{1}{2} \frac{A_{M-1}}{A_{j}}}\right) \geq-Q$,
there exists a unique root $\lambda^{\#}$ (here, $\left.-A_{M} / 4>\lambda^{\#} \geq-A_{M-1} / 4\right)$ such that $S_{M-1}\left(\lambda^{\#}\right)=-Q$ and hence we are done. The optimal solution $\mathcal{Q}^{\#}$ for Problem P1 is $\left\{\begin{array}{l}q_{j}^{\#}=-\frac{1}{a_{j}^{*}} \ln \left(-1-\frac{1-\sqrt{1+4 \lambda^{\# /} / A_{j}}}{2 \lambda^{\# / A}}\right) j=1,2, \ldots, M-1 ; \\ q_{M}^{\#}=0\end{array}\right.$

(ii) If $\max _{-A_{M} / 4>\lambda \geq-A_{M-1} / 4} S_{M-1}(\lambda)=$ $\sum_{j=1}^{M-1} \frac{1}{a_{j}^{*}} \ln \left(-1-\frac{1-\sqrt{1-A_{M-1} / A_{j}}}{-\frac{1}{2} \frac{A_{M-1}}{A_{j}}}\right)<-Q$, we have $\lambda<-A_{M-1} / 4$ and then from Eq.(12) we have

$$
\frac{A_{M-1} B_{M-1}}{\left(1+B_{M-1}\right)^{2}}+\lambda<\frac{A_{M-1}}{4}-\frac{A_{M-1}}{4}=0 ;
$$


that is,

$$
\frac{\partial L_{2}(\mathcal{Q}, \lambda)}{\partial q_{M-1}}<0
$$

Therefore,

$$
\sum_{j=1}^{M-1} \frac{v_{j} m_{j}^{*} p_{L T, j}}{1+e^{-a_{j}^{*} q_{j}}}+\lambda\left(\sum_{j=1}^{M-1} q_{j}-Q\right)
$$

is decreasing in $q_{M-1}$. Furthermore, it can be easily shown that

$$
\sum_{j=1}^{M-1} \frac{v_{j} m_{j}^{*} p_{L T, j} I\left(q_{j}>0\right)}{1+e^{-a_{j}^{*} q_{j}}}+\lambda\left(\sum_{j=1}^{M-1} q_{j}-Q\right)
$$

is decreasing in $q_{M-1}$. In order to maximize Eq.(23), let $q_{M-1}^{\#}=q_{M-1}=0$ and go to next step (the subsequent steps are similar to Step 3 to find $\ell^{\#}$ and a root $\lambda^{\#}$ such that $\left.S_{M-\ell \#}\left(\lambda^{\#}\right)=-Q\right)$.

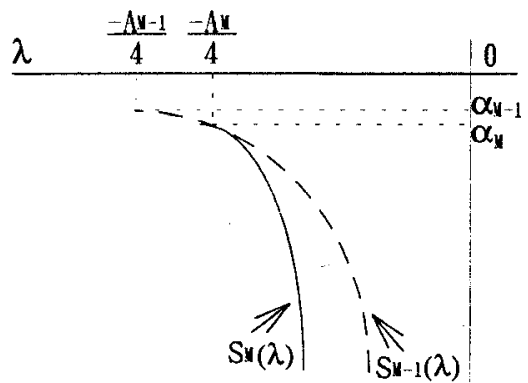

Figure 1. Functions of $S_{M}(\lambda)$ and $S_{M-1}(\lambda)$.

Based on the above description, we propose an efficient and novel optimization algorithm (called Algorithm 1) to determine the optimal solution for the resource allocation Problem P1.

\section{Algorithm 1:}

Step 1. Rearrange the indices of $A_{j}$ 's such that $A_{1} \geq$ $A_{2} \geq \ldots \geq A_{M} \geq A_{M+1}=0$ (here, $A_{M+1}=0$ is a dummy variable).

Step 2. Let $\ell=0$.

Step 3. If $\sum_{j=1}^{M-\ell} \frac{1}{a_{j}^{*}} \ln \left(-1-\frac{1-\sqrt{1-A_{M-\ell} / A_{j}}}{-A_{M-\ell} /\left(2 A_{j}\right)}\right) \geq-Q$, (let $\ell^{\#}=\ell$ ) there exists a unique root $\lambda^{\#}$ such that $S_{M-\ell \#}\left(\lambda^{\#}\right)=-Q$ and the optimal solution $\mathcal{Q}^{\#}$ is

$$
q_{j}^{\#}=-\frac{1}{a_{j}^{*}} \ln \left(-1-\frac{1-\sqrt{1+4 \lambda^{\#} / A_{j}}}{2 \lambda^{\#} / A_{j}}\right),
$$

$j=1,2, \ldots, M-\ell^{\#} ;$ otherwise, let $q_{M-\ell+1}^{\#}=$ $q_{M-\ell+1}=0$, set $\ell \leftarrow \ell+1$, and go to Step 3 .
In general, such linearly constrained minimization problem can be solved by the successive quadratic programming method based on the iterative formulation and solution of quadratic programming subproblems [16]. However, this method is complex. On the contrary, in Algorithm 1 the first $\ell^{\#}-1$ iterations just do simple numerical comparisons. In the $\ell^{\#}$ th iteration, since $S_{M-\ell \#}(\lambda)$ is decreasing in $\lambda$, there must exist a unique root $\lambda^{\#}$ such that $S_{M-\ell \#}\left(\lambda^{\#}\right)=-Q$ and hence the optimal solution $\mathcal{Q}^{\#}$ can be obtained. Note that $\lambda^{\#}$ can be easily obtained by simple numerical analysis. Therefore, Algorithm 1 is quite simple, efficient, and novel. By Lemma 1 it can be easily shown that this algorithm always converges in, at worst, $M-1$ steps. The value of the objective function given by Eq.(7) with the optimal solution $\mathcal{Q}^{\#}$ is

$$
\sum_{j=1}^{M} v_{j} m_{j}^{*}\left(1-\frac{p_{L T, j} I\left(q_{j}^{\#}>0\right)}{1+e^{-q_{j}^{\#}\left(a_{j} k+b_{j}\right)}}\right)
$$

\subsection{Minimizing the amount of testing re- sources}

In this subsection, we consider another resource allocation problem. Assuming that the number of software faults still undetected in the whole system after the application of $t_{1}, t_{2}, \ldots, t_{k}$ is less than or equal to $Z$. The project manager has to allocate an appropriate amount of testing resources to each module during the application of $t_{k}$ to minimize the total amount of testing resources required throughout module testing [2-4].

From Eq.(6), this resource allocation problem (called Problem P2) can be formulated as:

$$
\operatorname{Min} \sum_{j=1}^{M} q_{j}
$$

subject to

$$
\begin{aligned}
& \sum_{j=1}^{M} v_{j} m_{j}^{*}\left(1-\frac{p_{L T, j} I\left(q_{j}>0\right)}{1+e^{-q_{j}\left(a_{j} k+b_{j}\right)}}\right) \leq Z \\
& q_{j} \geq 0, j=1,2, \ldots, M
\end{aligned}
$$

Note that from Eq.(25) we have

$\sum_{j=1}^{M} \lim _{q_{j} \rightarrow \infty} v_{j} m_{j}^{*}\left(1 \frac{p_{L T, j} I\left(q_{j}>0\right)}{1+e^{-q_{j}\left(a_{j} k+b_{j}\right)}}\right)=\sum_{j=1}^{M} v_{j} m_{j}^{*}\left(1-p_{L T, j}\right)$.

That is, it is impossible to reduce the number of software faults still undetected in the whole system to $\sum_{j=1}^{M} v_{j} m_{j}^{*}\left(1-p_{L T, j}\right)$ after the application of test instance $t_{k}$ no matter which resource allocation method is used. This phenomenon is the limitation of the HGDM with logistic learning factor model. 
Ignoring the constraint $q_{j} \geq 0$ and using the Lagrange method [15], Problem $\mathrm{P} 2$ is equivalent to find the minimum of

$$
L_{3}(\mathcal{Q}, \lambda)=\sum_{j=1}^{M} q_{j}+\lambda\left(\sum_{j=1}^{M} v_{j} m_{j}^{*}\left(1-\frac{p_{L T, j} I\left(q_{j}>0\right)}{1+e^{-a_{j}^{*} q_{j}}}\right)-Z\right) .
$$

For convenience, we consider another problem (called Problem $P 2^{\prime}$ ):

$$
\operatorname{Min} \sum_{j=1}^{M} q_{j}
$$

subject to $\quad \sum_{j=1}^{M} v_{j} m_{j}^{*}\left(1-\frac{p_{L T, j}}{1+e^{-q_{j}\left(a_{j} k+b_{j}\right)}}\right) \leq Z$

$$
q_{j} \geq 0, j=1,2, \ldots, M \text {. }
$$

Ignoring the constraint $q_{j} \geq 0$ and using the Lagrange method [15], Problem $P 2^{\prime}$ is equivalent to find the minimum of

$$
L_{4}(\mathcal{Q}, \lambda)=\sum_{j=1}^{M} q_{j}+\lambda\left(\sum_{j=1}^{M} v_{j} m_{j}^{*}\left(1-\frac{p_{L T, j}}{1+e^{-a_{j}^{*} q_{j}}}\right)-Z\right) .
$$

The necessary conditions B1-B3 for a minimum of Eq. (27) to exist are as follows [15]:

$\mathrm{B} 1: \frac{\partial L_{4}(\mathcal{Q}, \lambda)}{\partial q_{j}}=0, j=1,2, \ldots, M$.

B2: $\lambda>0$.

B3: $\sum_{j=1}^{M} v_{j} m_{j}^{*}\left(1-\frac{p_{L T, j}}{1+e^{-a_{j}^{*} q_{j}}}\right)=Z$.

For Condition B1, from Eq.(27) we have

$$
\begin{aligned}
\frac{\partial L_{4}(\mathcal{Q}, \lambda)}{\partial q_{j}} & =1-\lambda \frac{v_{j} m_{j}^{*} p_{L T, j} a_{j}^{*} e^{-a_{j}^{*} q_{j}}}{\left(1+e^{-a_{j}^{*} q_{j}}\right)^{2}} \\
& =1-\lambda \frac{A_{j} B_{j}}{\left(1+B_{j}\right)^{2}}=0, j=1,2, \ldots, M(
\end{aligned}
$$

Since $q_{j}$ should be larger than or equal to zero, we have $0<B_{j} \leq 1$ for $j=1,2, \ldots, M$. Therefore, from Eq.(29) we have $\lambda>0$ (i.e., Condition B2 is satisfied) and

$$
\frac{B_{j}}{\left(1+B_{j}\right)^{2}}=\frac{1}{\lambda A_{j}} \text {. }
$$

Since $0<B_{j} \leq 1$, from Eq.(30) we can easily obtain

$$
\lambda \geq \frac{4}{A_{j}}, j=1,2, \ldots, M,
$$

and the root of Eq.(30) is

$$
B_{j}=-1-\frac{1-\sqrt{1+4 \lambda_{j}^{*}}}{2 \lambda_{j}^{*}},
$$

where $\lambda_{j}^{*}=-1 /\left(\lambda A_{j}\right)$. Therefore, we have

$$
q_{j}=-\frac{1}{a_{j}^{*}} \ln \left(-1+\frac{1-\sqrt{1-4 /\left(\lambda A_{j}\right)}}{2 /\left(\lambda A_{j}\right)}\right), j=1,2, \ldots, M .
$$

Let

$$
\begin{aligned}
R_{L}(\lambda) & =\sum_{j=1}^{L} v_{j} m_{j}^{*}\left(1-\frac{p_{L T, j}}{1+e^{-a_{j}^{*} q_{j}}}\right) \\
& =\sum_{j=1}^{L} v_{j} m_{j}^{*}\left(1-p_{L T, j} \frac{2 /\left(\lambda A_{j}\right)}{1-\sqrt{1-4 /\left(\lambda A_{j}\right)}}\right) \\
& =\sum_{j=1}^{L} r_{j}(\lambda), L=1,2, \cdots, M,
\end{aligned}
$$

where

$$
r_{j}(\lambda)=v_{j} m_{j}^{*}\left(1-p_{L T, j} \frac{2 /\left(\lambda A_{j}\right)}{1-\sqrt{1-4 /\left(\lambda A_{j}\right)}}\right) .
$$

By simple calculation, we have the following lemma.

\section{Lemma 2.}

(i) $r_{j}(\lambda)$ is decreasing in $\lambda$;

(ii) $\lim _{\lambda \rightarrow \infty} r_{j}(\lambda)=v_{j} m_{j}^{*}\left(1-p_{\iota T, j}\right)$;

(iii) $\lim _{\lambda \rightarrow 4 / A_{i}} r_{j}(\lambda)=v_{j} m_{j}^{*}\left(1-\frac{1}{2 p_{L T, j}}\right)$, where $r_{j}(\lambda)$ is given in $\mathrm{Eq} .(35)$.

Since $\lambda \geq 4 / A_{j}$ for $j=1,2, \ldots, M$, for convenience we rearrange the indices of $A_{j}$ 's such that $A_{1} \geq A_{2} \geq$ $\ldots \geq A_{M}$. By Lemma 2 we have

$$
\begin{gathered}
\sum_{j=1}^{M} v_{j} m_{j}^{*}\left(1-p_{L T, j}\right) \leq R_{M}(\lambda) \leq \\
\sum_{j=1}^{M} v_{j} m_{j}^{*}\left(1-p_{L T, j} \frac{A_{M} /\left(2 A_{j}\right)}{1-\sqrt{1-A_{M} / A_{j}}}\right) .
\end{gathered}
$$

Since $R_{L}(\lambda)=\sum_{j=1}^{L} r_{j}(\lambda)$ and $r_{j}(\lambda)$ is decreasing in $\lambda, R_{L}(\lambda)$ is decreasing in $\lambda$ for $L=1,2, \cdots, M$. Furthermore, from Eq.(35) we have $r_{j}(\lambda)>0$ for all $j$, and from Eq. (36) $R_{M}(\lambda)$ and $R_{M-1}(\lambda)$ are shown in Figure 2, where $\alpha_{M}=\sum_{j=1}^{M} v_{j} m_{j}^{*}(1-$ $\left.p_{L T, j} \frac{A_{M} /\left(2 A_{j}\right)}{1-\sqrt{1-A_{M} / A_{j}}}\right), \quad \alpha_{M-1}=\sum_{j=1}^{M-1} v_{j} m_{j}^{*}(1-$ $\left.p_{L T, j} \frac{A_{M-1} /\left(2 A_{j}\right)}{1-\sqrt{1-A_{M-1} / A_{j}}}\right), \beta_{M}=\sum_{j=1}^{M} v_{j} m_{j}^{*}\left(1-p_{L T, j}\right)$, and $\beta_{M-1}=\sum_{j=1}^{M-1} v_{j} m_{j}^{*}\left(1-p_{L T, j}\right)$. To satisfy the 
constraint $\sum_{j=1}^{M} v_{j} m_{j}^{*}\left(1-p_{L T, j} /\left(1+e^{-a_{j}^{*} q_{j}}\right)\right)=Z$ (i.e., Condition B3) and based on Figure 2, the main steps for finding the minimum of Problem P2 are depicted in the following:

Step 1. Rearrange the indices of $A_{j}$ 's such that $A_{1} \geq$ $A_{2} \geq \ldots \geq A_{M}$.

Step 2. (i) If $\sum_{j=1}^{M} v_{j} m_{j}^{*}\left(1-p_{L T, j}\right) \geq Z$, it is impossible to reduce the number of still undetected software faults to $Z$ after the application of test instance $t_{k}$.

(ii) If $\sum_{j=1}^{M} v_{j} m_{j}^{*}\left(1-p_{L T, j}\right)<Z \leq$ $\sum_{j=1}^{M} v_{j} m_{j}^{*}\left(1-p_{L T, j} \frac{A_{M} /\left(2 A_{j}\right)}{1-\sqrt{1-A_{M} / A_{j}}}\right)$, there exists a unique root $\lambda^{\#}$ (here, $\lambda^{\#} \geq 4 / A_{M}$ ) such that $R_{M}\left(\lambda^{\#}\right)=Z$ and hence we are done. The optimal solution $\mathcal{Q}^{\#}$ for Problem $\mathrm{P} 2$ is

$q_{j}^{\#}=-\frac{1}{a_{j}^{*}} \ln \left(-1 \frac{1-\sqrt{1-4 /\left(\lambda^{\#} A_{j}\right)}}{2 /\left(\lambda^{\#} A_{j}\right)}\right) j=1,2, \ldots, M$.

Since $\left\{\left(q_{1}, q_{2}, \ldots, q_{M}\right):\left(q_{1}, q_{2}, \ldots, q_{M}\right)\right.$ satisfies Eq.(25)\} is the subset of $\left\{\left(q_{1}, q_{2}, \ldots, q_{M}\right)\right.$ : $\left(q_{1}, q_{2}, \ldots, q_{M}\right)$ satisfies Eq. $\left.(26)\right\}$ and $q_{j}^{\#}>$ $0, j=1,2, \ldots, M$, the optimal solution for Problem P2 is also $\mathcal{Q}^{\#}$

(iii) If $\sum_{j=1}^{M} v_{j} m_{j}^{*}\left(1-p_{L T, j} \frac{A_{M} /\left(2 A_{j}\right)}{1-\sqrt{1-A_{M} / A_{j}}}\right)<Z$, we have $\lambda<4 / A_{M}$ and hence from Eq.(29) we have

$1-\lambda \frac{A_{M} B_{M}}{\left(1+B_{M}\right)^{2}} \geq 1-\frac{4}{A_{M}} \frac{A_{M} B_{M}}{\left(1+B_{M}\right)^{2}} \geq 0$

that is,

$$
\frac{\partial L_{4}(\mathcal{Q}, \lambda)}{\partial q_{M}}>0 \text {. }
$$

Therefore, $L_{4}(\mathcal{Q}, \lambda)$ is increasing in $q_{M}$. Furthermore, it can be easily shown that $L_{3}(\mathcal{Q}, \lambda)$ is also increasing in $q_{M}$. To minimize $L_{3}(\mathcal{Q}, \lambda)$, let $q_{M}^{\#}=q_{M}=0$ and go to Step 3.

Step 3. (i) If $\sum_{j=1}^{M-1} v_{j} m_{j}^{*}\left(1-p_{L T, j}\right) \geq Z-v_{M} m_{M}^{*}$, it is impossible to reduce the number of stil1 undetected software faults to $Z$ after the application of test instance $t_{k}$.

(ii) If $\sum_{j=1}^{M-1} v_{j} m_{j}^{*}\left(1-p_{L T, j}\right)<Z-v_{M} m_{M}^{*} \leq$ $\sum_{j=1}^{M-1} v_{j} m_{j}^{*}\left(1-p_{L T, j} \frac{A_{M-1} /\left(2 A_{j}\right)}{1-\sqrt{1-A_{M-1} / A_{j}}}\right)$

there exists a unique root $\lambda^{\#}$ (here, $4 / A_{M}>$ $\left.\lambda^{\#} \geq 4 / A_{M-1}\right)$ such that $R_{M-1}\left(\lambda^{\#}\right)=Z-$
$v_{M} m_{M}^{*}$ and hence we are done. The optimal solution $\mathcal{Q}^{\#}$ for Problem P2 is

$\left\{\begin{array}{l}q_{j}^{\#}=-\frac{1}{a_{j}^{*}} \ln \left(-1+\frac{\sqrt{1-4 /\left(\lambda^{\#} A_{j}\right)}}{2 /\left(\lambda^{\#} A_{j}\right)}\right) j=1,2, \ldots, M-1 ; \\ q_{M}^{\#}=0,\end{array}\right.$

(iii) If $\sum_{j=1}^{M-1} v_{j} m_{j}^{*}\left(1-p_{L T, j} \frac{A_{M-1} /\left(2 A_{j}\right)}{1-\sqrt{1-A_{M-1} / A_{j}}}\right)<$ $Z-v_{M} m_{M}^{*}$, we have $\lambda<4 / A_{M-1}$ and then from Eq.(29) we have

$1-\lambda \frac{A_{M-1} B_{M-1}}{\left(1+B_{M-1}\right)^{2}} \geq 1-\frac{4}{A_{M-1}} \frac{A_{M-1} B_{M-1}}{\left(1+B_{M-1}\right)^{2}} \geq 0 ;$

that is,

$$
\frac{\partial L_{4}(\mathcal{Q}, \lambda)}{\partial q_{M-1}}>0
$$

Therefore,

$$
\begin{gathered}
\sum_{j=1}^{M-1} q_{j}+\lambda\left\{\sum_{j=1}^{M-1} v_{j} m_{j}^{*}\left(1-\frac{p_{L T, j}}{1+e^{-a_{j}^{*} q_{j}}}\right)\right\} \\
-\lambda\left(Z-v_{M} m_{M}^{*}\right)
\end{gathered}
$$

is increasing in $q_{M-1}$. Furthermore, it can be easily shown that

$$
\begin{aligned}
& \sum_{j=1}^{M-1} q_{j}+\lambda\left\{\sum_{j=1}^{M-1} v_{j} m_{j}^{*}\left(1-\frac{p_{L T, j} I\left(q_{j}>0\right)}{1+e^{-a_{j}^{*} q_{j}}}\right)\right\} \\
& -\lambda\left(Z-v_{M} m_{M}^{*}\right)
\end{aligned}
$$

is increasing in $q_{M-1}$. To minimize Eq.(38), let $q_{M-1}^{\#}=q_{M-1}=0$ and go to next step (the subsequent steps are similar to Step 3 ).

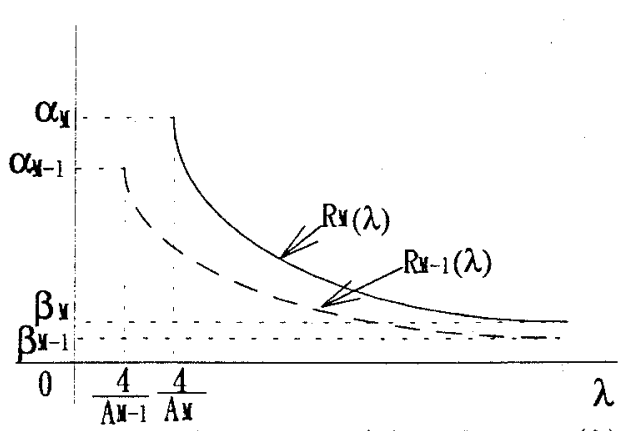

Figure 2. Functions of $R_{M}(\lambda)$ and $R_{M-1}(\lambda)$.

Based on above description, we propose an efficient and novel optimization algorithm (called Algorithm 2) to determine the optimal solution for the resource allocation Problem P2.

\section{Algorithm 2:}

Step 1. Rearrange the indices of $A_{j}$ 's such that $A_{1} \geq$ $A_{2} \geq \ldots \geq A_{M}$. 
Step 2. Let $\ell=0$ and $Z^{*}=0$.

Step 3. (i) If $\sum_{j=1}^{M-\ell} v_{j} m_{j}^{*}\left(1-p_{L T, j}\right) \geq Z-Z^{*}$, it is impossible to reduce the number of still undetected software faults to $Z$ after the application of test instance $t_{k}$.

(ii) If $\sum_{j=1}^{M-\ell} v_{j} m_{j}^{*}\left(1-p_{L T, j}\right)<Z-Z^{*} \leq$ $\sum_{j=1}^{M-\ell} v_{j} m_{j}^{*}\left(1-p_{L T, j} \frac{A_{M-\ell} /\left(2 A_{j}\right)}{1-\sqrt{1-A_{M-\ell} / A_{j}}}\right)$, (letting $\ell^{\#}=\ell$ ) there exists a unique root $\lambda^{\#}$ such that $R_{M-\ell *}\left(\lambda^{\#}\right)=Z-Z^{*}$ and the optimal solution $\mathcal{Q}^{\#}$ is

$q_{j}^{\#}=-\frac{1}{a_{j}^{*}} \ln \left(-1 \frac{1-\sqrt{1-4 /\left(\lambda^{\#} A_{j}\right)}}{2 /\left(\lambda^{\#} A_{j}\right)}\right) j=1,2, \ldots, M \ell^{\#}$; otherwise, let $q_{M-\ell+1}^{\#}=q_{M-\ell+1}=0$, set $Z^{*} \leftarrow Z^{*}+v_{M-\ell} m_{M-\ell}^{*}$, set $\ell \leftarrow \ell+1$, and go to Step 3 .

In Algorithm 2 the first $\ell^{\#}-1$ iterations just do simple numerical comparisons. In the $\ell^{\#}$ th iteration, since $R_{M-\ell \#}(\lambda)$ is decreasing in $\lambda$, there must exist a unique root $\lambda^{\#}$ such that $R_{M-\ell \#}\left(\lambda^{\#}\right)=Z-Z^{*}$ and hence the optimal solution $\mathcal{Q}^{\#}$ can be obtained. Note that $\lambda^{\#}$ can be easily obtained by simple numerical analysis. Similar to the discussion in Subsection 3.1, Algorithm 2 is, therefore, also quite simple, efficient, and novel. Furthermore, by Lemma 2 it can be easily shown that this algorithm always converges in, at worst, $M-1$ steps. The value of the objective function given by Eq.(25) with the optimal solution $\mathcal{Q}^{\#}$ is

$$
\sum_{j=1}^{M} q_{j}^{\#}=\sum_{j=1}^{M-\ell^{\#}}-\frac{1}{a_{j}^{*}} \ln \left(-1+\frac{1-\sqrt{1-4 /\left(\lambda^{\#} A_{j}\right)}}{2 /\left(\lambda^{\#} A_{j}\right)}\right)
$$

\section{Relationship between Optimal, Average, and Proportional Allocation Methods}

In Section 3.1, we discuss the problem of optimal resource allocation if the total amount of testing resources for module testing is specified. In this section, based on the concepts of average allocation and proportional allocation, we introduce two simple resource allocation methods, the average allocation method and the proportional allocation method, respectively. Furthermore, we also investigate the relationship between these three allocation methods.

A resource allocation method is called an average allocation method if the testing resources are allocated to each module evenly. Let $q_{j, a v g}$ be the amount of testing resources allocated to module $j$ during the application of $t_{k}$ by the average allocation method, and then we have

$$
q_{j, a v g}=\frac{Q}{M}, j=1,2, \ldots, M
$$

Let $Z_{\text {avg }}$ be the number of still undetected faults in the whole system after the application of $t_{k}$ by the average allocation method, and then from Eq.(6) we have

$$
Z_{a v g}=\sum_{j=1}^{M} v_{j} m_{j}^{*}\left(1-\frac{p_{L T, j} I\left(q_{j, a v g}>0\right)}{1+e^{-q_{j, a v g} a_{j}^{*}}}\right) .
$$

A resource allocation method is called a proportional allocation method if the amount of testing resources allocated to module $j$ is proportional to the number of still undetected faults in module $j$. Let $q_{j, \text { prop }}$ be the amount of testing resources allocated to module $j$ during the application of $t_{k}$ by the proportional allocation method, and then we have $q_{j, p r o p}$

$$
q_{j, \text { prop }}=Q \frac{m_{j}^{*}}{\sum_{i=1}^{M} m_{i}^{*}}, j=1,2, \ldots, M .
$$

Note that if $m_{1}^{*}=m_{2}^{*}=\ldots=m_{M}^{*}$, the proportional allocation method is equivalent to the average allocation method. Let $Z_{\text {prop }}$ be the number of still undetected faults in the whole system after the application of $t_{k}$ by the proportional allocation method, and then from Eq.(6) we have

$$
Z_{\text {prop }}=\sum_{j=1}^{M} v_{j} m_{j}^{*}\left(1-\frac{p_{L T, j} I\left(q_{j, p r o p}>0\right)}{1+e^{-q_{j, p r o p} a_{j}^{*}}}\right) .
$$

The optimal allocation method is said to be better than the average and the proportional methods since $Z_{\text {opt }} \leq Z_{\text {avg }}$ and $Z_{\text {opt }} \leq Z_{\text {prop. }}$. However, we are also interested in how much better the optimal allocation method is. In the following, we investigate the value of $Z_{\text {opt }} / Z_{\text {prop }}$ under the case $p_{L T, j}=p_{L T}$ for $j=1,2, \ldots, M$.

From Eqs.(24) and (43), we have

$$
\begin{gathered}
g\left(p_{L T}\right)=\frac{Z_{o p t}}{Z_{\text {prop }}} \\
=\frac{\sum_{j=1}^{M} v_{j} m_{j}^{*}\left(1-p_{L T} I\left(q_{j}^{\#}>0\right) /\left(1+e^{-q_{j}^{\#} a_{j}^{*}}\right)\right)}{\sum_{j=1}^{M} v_{j} m_{j}^{*}\left(1-p_{L T} I\left(q_{j, p r o p}>0\right) /\left(1+e^{-q_{j, p r a p} a_{j}^{*}}\right)\right)} .
\end{gathered}
$$

With the fact of $Z_{\text {opt }} \leq Z_{\text {prop }}$ and some manipulations, we have

$$
\frac{\partial g\left(p_{L T}\right)}{\partial p_{L T}} \leq 0
$$

That is, $Z_{\text {opt }} / Z_{\text {prop }}$ is decreasing in $p_{L T}$, which mean$s$ that the larger the $p_{L T}$ is, the smaller the ratio of the number of still undetected faults by the optimal allocation method to that by the proportional method 
is. In other words, as $p_{L T}$ increases, the optimal allocation method is much better than the proportional allocation method. Furthermore, since $p_{L T}$ represents the skill of detecting faults [12], from Eq.(44) the following can be observed: the better the skill of detecting faults, the better the optimal allocation method (compared with the proportional allocation method).

By the same argument on $Z_{\text {opt }} / Z_{\text {prop }}$, we have the property that $Z_{\text {opt }} / Z_{\text {prop }}$ is decreasing in $p_{L T}$. That is, the better the skill of detecting faults, the better the optimal allocation method (compared with the average allocation method).

\section{Numerical Examples}

Consider a software system consisting of 5 modules (i.e., $M=5$ ). Suppose there are 4 test instances $t_{1}, t_{2}, t_{3}, t_{4}$ (i.e., $k=5$ ) applied during module testing so far. For each module, the parameters $a_{j}, b_{j}$, $m_{j}$, and $p_{L T, j}, j=1,2, \ldots, 5$ can be estimated by using the software failure data, and then the estimated value of $m_{j}^{*}$ can be obtained. The estimated values of $m_{j}^{*}, a_{j}, b_{j}, p_{L T, j}$ and the weighting factor $v_{j}$ are assumed and listed in Table 1 . Consequently, the total number of still undetected faults after the application of $t_{1}, t_{2}, t_{3}, t_{4}$ is assumed to be 200 (since $\left.\sum_{j=1}^{5} m_{j}^{*}=200\right)$.

\section{Example 1. Minimizing the total number of undetected faults}

Suppose the total amount of testing resources $Q$ for module testing during the application of $t_{5}$ is $20 k$ (i.e., $20,000)$ man-hours. The manager has to allocate the $20 \mathrm{k}$ man-hours to the 5 modules to minimize the total number of still undetected faults after the application of $t_{5}$. Using the values of $m_{j}^{*}, a_{j}, b_{j}, p_{L T, j}$, and $v_{j}$ in Table 1 , the optimal solutions $q_{j}^{\#}$ estimated by Algorithm 1 are shown in Table 1 . Consequently, the total number of still undetected software faults after the application of $t_{5}$ estimated by Eq.(24) is $Z_{\text {opt }}=115$. That is, the total number of still undetected faults is anticipated to be reduced from 200 to 115 by using the testing resources of $20 k$ man-hours. The reduction in the undetected software faults is about $42.5 \%$.

Table 1. Allocated Testing Resources $q_{j}^{\#}$ for Minimizing undetected Software Faults.

( $Q$ is assumed to be $20 k$ man-hours)

\begin{tabular}{cccccccc}
\hline Module & $m_{j}^{*}$ & $a_{j}$ & $b_{j}$ & $p_{L T, j}$ & $v_{j}$ & $q_{j}^{\#}$ & $z_{j}$ \\
\hline 1 & 50 & 0.02 & 0.1 & 1.0 & 1 & 12.79 & 3.6 \\
2 & 45 & 0.08 & 0.2 & 0.6 & 1 & 5.17 & 19.2 \\
3 & 40 & 0.2 & 1 & 0.3 & 1 & 1.76 & 28.3 \\
4 & 35 & 0.8 & 5 & 0.03 & 1 & 0.28 & 34 \\
5 & 30 & 2.0 & 10 & 0.003 & 1 & 0 & 30 \\
\hline \multicolumn{6}{c}{$Z_{\text {opt }}=\sum_{j=1}^{5} z_{j}=115$ (42.5\% reduction) }
\end{tabular}

Based on the average allocation method, from $\mathrm{E}$ q. (40) we have $q_{1, a v g}=q_{2, a v g}=\ldots=q_{5, a v g}=4$ and then from Eq.(41) we have $Z_{a v g}=128$. Furthermore, based on the proportional allocation method, from $\mathrm{E}$ q.(42) we have $q_{1, p r o p}=5, q_{2, \text { prop }}=4.5, q_{3, \text { prop }}=4$, $q_{4, \text { prop }}=3.5$, and $q_{5, \text { prop }}=3$. Therefore, from Eq. (43) we have $Z_{\text {prop }}=125$. Obviously, the optimal allocation method is better than the proportional method and the average method. Furthermore, the optimal resource allocation method is very efficient in this example since the difference of $Z_{\text {prop }}-Z_{\text {opt }}$ is significant.

In the following, consider the case $p_{L T, j}=p_{L T}$ for $j=1,2, \ldots, 5$ and the estimated values of $m_{j}^{*}, a_{j}$, $b_{j}$ and $v_{j}$ are also listed in Table 1. The curves of $Z_{\text {opt }} / Z_{\text {prop }}$ and $Z_{\text {opt }} / Z_{\text {avg }}$ vs. $p_{L T}$ are depicted in Figure 3. As $p_{L T}=0.1$, from Figure 3 we have $Z_{\text {opt }} / Z_{\text {prop }}=0.995$ and $Z_{\text {opt }} / Z_{\text {avg }}=0.993$. As $p_{L T}=0.9$, we have $Z_{\text {opt }} / Z_{\text {prop }}=0.651$ and $Z_{\text {opt }} / Z_{\text {avg }}=0.586$. Obviously, if $p_{L T}$ is smaller, the differences of the number of still undetected software faults among these three resource allocation methods are less significant. Therefore, the average resource allocation method can be employed due to its simplicity if $p_{L T}$ is smaller. On the contrary, if $p_{L T}$ is larger, the optimal resource allocation method should be applied since it reduces the number of still undetected software faults much more significantly. In summary, both curves in Figure 3 are decreasing in $p_{L T}$; that is, the larger the $p_{L T}$, the better the optimal allocation method (compared with the average and the proportional allocation methods).

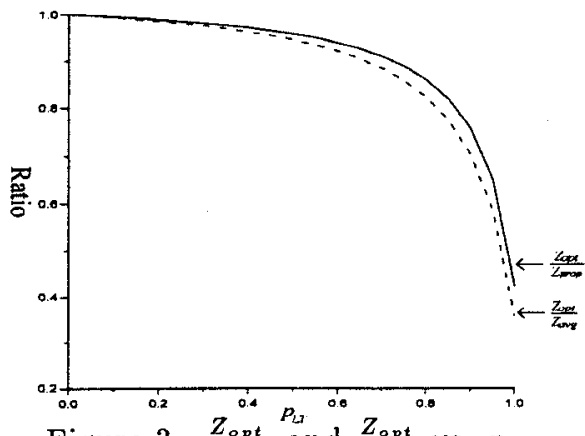

Figure 3. $\frac{Z_{\text {opt }}}{Z_{\text {prop }}}$ and $\frac{Z_{\text {opt }}}{Z_{a v g}}$ vs. $p_{L T}$.

Figure 3 also indicates that the proportional allocation method is better than the average allocation method since $Z_{\text {opt }} / Z_{\text {prop }} \geq Z_{\text {opt }} / Z_{\text {avg }}$ for $0<p_{L T} \leq 1$. This result meets our intuition.

\section{Example 2. Minimizing the total amount of testing resources}

Suppose the number of still undetected software faults after the application of $t_{5}$ needs to be reduced from 200 to 120 . The manager has to determine how much testing resource is to be allocated for each module to minimize the total amount of testing resources. Using the values of $m_{j}^{*}, a_{j}, b_{j}, p_{L T, j}$, and $v_{j}$ in Table 
1, the optimal solutions $q_{j}^{\#}$ estimated by Algorithm 2 are shown in Table 2. Consequently, the total amoun$\mathrm{t}$ of testing resources in module testing estimated by Eq. (39) is $14.7 k$ man-hours. That is, the amount of testing resources spent during the application of $t_{5}$ is anticipated to be $14.7 k$ man-hours.

Table 2. Allocated Testing Resources $q_{j}^{\#}$ for Minimizing Total Testing Resources.

\begin{tabular}{ccc}
\multicolumn{3}{c}{$(Z$ is assumed to be 120$)$} \\
\hline Module & $q_{j}^{\#}$ & $z_{j}$ \\
\hline 1 & 9.03 & 7.1 \\
2 & 4.04 & 20.2 \\
3 & 1.44 & 28.6 \\
4 & 0.19 & 34.1 \\
5 & 0.00 & 30 \\
\hline$\sum_{j=1}^{5} q_{j}^{\#}=14.7 k$ man-hours
\end{tabular}

\section{Conclusions}

In this paper, based on the HGDM with logistic learning factor, we investigate two optimal resource allocation problems in software module testing: 1) minimization of the number of software faults still undetected in the system after testing given the total amount of testing resources and 2) minimization of the total amount testing resources required given the number of software faults still undetected in the system after testing. Two efficient and novel optimization algorithms based on the concept of Lagrange multiplier method for the above two problems are proposed, respectively. In addition, the relationship between the optimal, average, and proportional resource allocation methods is investigated. Experimental results show that the optimal allocation methods are very efficien$\mathrm{t}$ for solving the testing resource allocation problem. Furthermore, the results show that the better the skill of detecting faults, the better the optimal allocation method.

Acknowledgment. We would like to express our gratitude for the support of the National Science Council, Taiwan, R.O.C., under Grants NSC85-2221E002-015. Reviewers' comments are also highly appreciated.

\section{References}

[1] M. V. Zelkowitz, "Perspectives of software engineering," ACM Computing Surveys, Vol. 10, pp. 197-216, 1978.

[2] P. Kubat and H. S. Koch, "Managing testprocedures to achieve reliable software," IEEE Trans. on Reliability, Vol. 32, No. 3, pp. 299-303, 1983.

[3] H. Ohtera and S. Yamada, "Optimal allocation \& control problems for software-testing resources,"
IEEE Trans. on Reliability, Vol. 39, No. 2, pp. 171-176, 1990.

[4] Y. W. Leung, "Software reliability growth model with debugging efforts," Microelectron. Reliab., Vol. 32, No. 5, pp. 699-704, 1992.

[5] J. D. Musa, A. Iannino, and K. Okumoto, Software Reliability - Measurement, Prediction, Application, McGraw-Hill, New York, 1987.

[6] M. Xie, Software Reliability Modeling, World Scientific Publishing Company, Singapore, 1991.

[7] M. R. Lyu (ed.), Handbook of Software Reliability Engineering, McGraw-Hill, New York, 1996.

[8] Y. Tohma, K. Tokunaga, S. Nagase, and Y. Murata, "Structural approach to the estimation of the number of residual software faults based on the hyper-geometric distribution," IEEE Trans. on Software Engineering, vol. 15, No. 3, pp. 345355, March 1989.

[9] Y. Tohma, H. Yamano, M. Ohba, and R. Jacoby, "The estimation of parameters of the hypergeometric distribution and its application to the software reliability growth model," IEEE Trans. on Software Engineering, vol. SE-17, No. 5, pp. 483-489, May 1991.

[10] R. Jacoby and Y. Tohma, "Parameter value computation by least square method and evaluation of software availability and reliability at service-operation by the hyper-geometric distribution software reliability growth model (HGDM)," Proc. 13th Int. Conf. Software Engineering, pp. 226-237, 1991.

[11] T. Minohara and Y. Tohma, "Parameter estimation of hyper-geometric distribution software reliability growth model by genetic algorithms," Proc. 6th Int. Symposium on Software Reliability Engineering, pp. 324-329, 1995.

[12] R. H. Hou, S. Y. Kuo, and Y. P. Chang, "Applying various learning curves to hyper-geometric distribution software reliability growth model," Proc. 5th Int. Symposium on Software Reliability Engineering, pp. 7-16, 1994.

[13] R. H. Hou, S. Y. Kuo, and Y. P. Chang, "Optimal release times for software systems with scheduled delivery time based on the HGDM " IEEE Trans. on Computers (accepted for publication).

[14] R. H. Hou, S. Y. Kuo, and Y. P. Chang, "Optimal release policy for hyper-geometric distribution software reliability growth model," IEEE Trans. on Reliability (accepted for publication).

[15] M. S. Bazaraa and C. M. Shetty, Nonlinear Programming: Theory and Algorithms, Wiley, New York, 1993.

[16] M. J. D. Powell, A tolerant algorithm for linearly constrained optimization calculations, DAMTP Report NA17, University of Cambridge, England, 1988. 